\title{
Moving deforming mesh technique for fluid flow simulation with immersed boundary conditions
}

\author{
Vladimir Garanzha*, Liudmila Kudryavtseva*† and Valeria Tsvetkova ${ }^{\dagger}$ \\ * Dorodnicyn Computing Center FRC CSC RAS \\ 119333, Vavilova str. 40, Moscow, Russia \\ e-mail: garan@ccas.ru - Web page http://www.ccas.ru/gridgen/lab \\ † Keldysh Institute of Applied Mathematics RAS \\ 125047, Miusskaya sq.4, Moscow, Russia
}

\begin{abstract}
We suggest an algorithm for generation of a moving adaptive mesh for a system of moving bodies defined by the distance-like implicit function. The objective is to improve accuracy of the immersed boundary conditions for viscous flow solver NOISETTE [1]. Time-dependent control metric is constructed on top of the implicit function, and minimization of quasi-isometric mesh quality functional [2] is used to make the mesh follow the metric. We expect that mesh compression zone would travel in the computational domain and would precisely follow the boundary of bodies. For efficient parallel execution of NOISETTE solver it is required that general unstructured $2 \mathrm{~d}$ or $3 \mathrm{~d}$ mesh is deformed without connectivity changes, which makes mesh adaptation problems quite hard.

We use the preconditioned gradient search technique for the mesh quality functional in order to compute displacements of each mesh vertex. It is too expensive to apply minimization at each time step until convergence. So we use special euristic predictor-interpolation scheme. A predictor is large mesh displacement which is computed by solving linear system with approximate Hessian matrix of the mesh functional twice. This predictor provides admissible deformation for a time step exceeding that of the flow solver, hence we can use simple linear interpolation in time in order to compute mesh in the intermediate time levels. We prove that all interpolates between the initial and the displaced states are nonsingular deformations of the initial mesh and can be used for numerical simulations with small time steps, which greatly improves the efficiency of the remeshing algorithm. We compute space-time mesh deformation trajectories in such a way that each space-time beam, i.e., trajectory of single mesh cell consists of nondegenerate cells.

The main problem that we encounter is elimination of oscillations in the trajectories. We identified the potential source of oscillations: besides true time step we should also use the best scaling factor along minimization direction which also has the meaning of a time step but in fact it is not.

The euristic approach which experimentally provides the smoothest behaviour of the mesh trajectories is based on two linear solves per predictor computation and resembles the two-stage Runge-Kutta scheme for certain artificial PDE. To be more precise, we use $2 d$ linear solves in $\mathbb{R}^{d}$ with linear systems corresponding to FE approximations of scalar Laplace-like equations per several (say 5-10) flow solver time steps, hence resulting moving mesh technique becomes competitive to mesh movement technique based on Laplace-like linear equations. However, unlike linear mesh solvers, the presented algorithm does not impose any limitation on the domain shape and the mesh elements type. It can be applied in the case of multiple dimensions and for high-order elements.

Due to the advanced parallel linear solver, a reasonable parallel scalability of the numerical algorithm was demonstrated.

We investigate relations between mesh compression and anisotropy in the layer, smoothness measures of the mesh cell trajectories and viscous flow charactrictics, in particular drag and lift coefficients.
\end{abstract}




\section{REFERENCES}

[1] Abalakin, I., Bakhvalov, P., Kozubskaya, T. Edge-based reconstruction schemes for unstructured tetrahedral meshes. Int. J. Num. Meth. Fluids, Vol. 81 No .6, pp. 331-356, (2016).

[2] Garanzha, V.A., Kudryavtseva, L.N., Utyzhnikov, S.V. Untangling and optimization of spatial meshes. J. of Comp. Appl. Math. 269, 24-41 (2014).

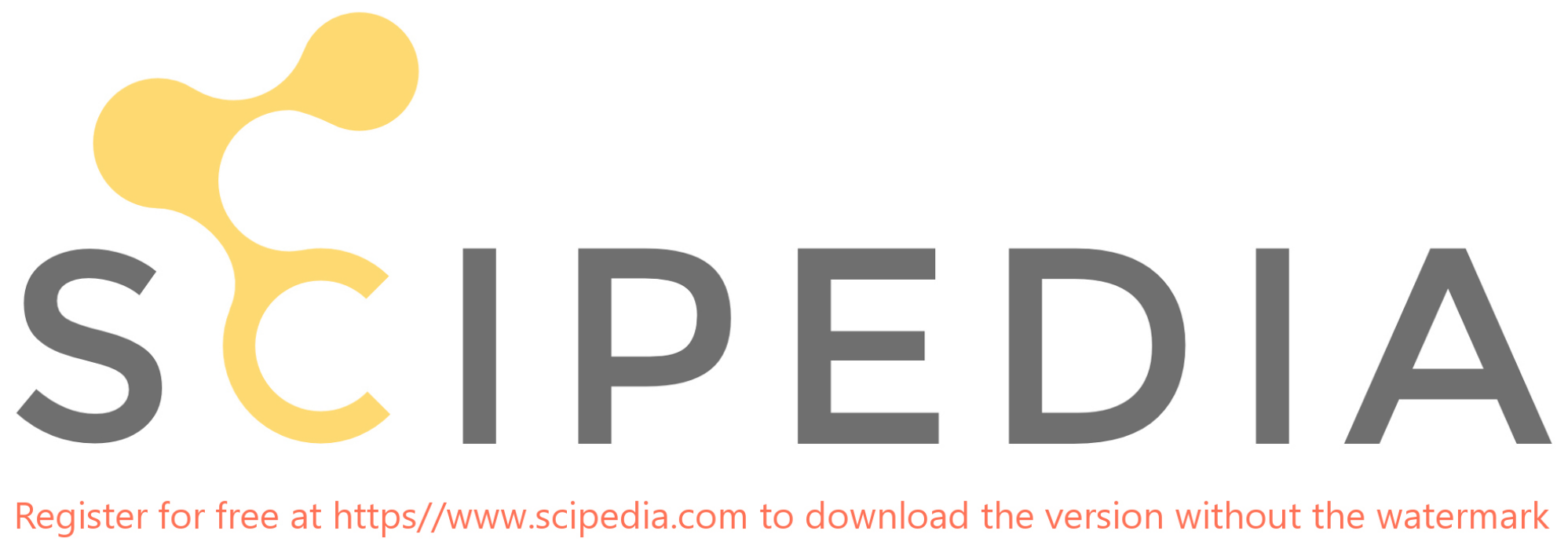

